\title{
Robustness of IPSA optimized high-dose-rate prostate brachytherapy treatment plans to catheter displacements
}

\author{
Joel Poder, MScl,2, May Whitaker, MScl \\ IRadiation Oncology, Chris O’Brien Lifehouse, Camperdown, NSW, ${ }^{2}$ Centre for Medical Radiation Physics, University of Wollongong, \\ Wollongong, NSW, Australia
}

\begin{abstract}
Purpose: Inverse planning simulated annealing (IPSA) optimized brachytherapy treatment plans are characterized with large isolated dwell times at the first or last dwell position of each catheter. The potential of catheter shifts relative to the target and organs at risk in these plans may lead to a more significant change in delivered dose to the volumes of interest relative to plans with more uniform dwell times.

Material and methods: This study aims to determine if the Nucletron Oncentra dwell time deviation constraint (DTDC) parameter can be optimized to improve the robustness of high-dose-rate (HDR) prostate brachytherapy plans to catheter displacements. A set of 10 clinically acceptable prostate plans were re-optimized with a DTDC parameter of 0 and 0.4 . For each plan, catheter displacements of 3, 7, and $14 \mathrm{~mm}$ were retrospectively applied and the change in dose volume histogram (DVH) indices and conformity indices analyzed.

Results: The robustness of clinically acceptable prostate plans to catheter displacements in the caudal direction was found to be dependent on the DTDC parameter. A DTDC value of 0 improves the robustness of planning target volume (PTV) coverage to catheter displacements, whereas a DTDC value of 0.4 improves the robustness of the plans to changes in hotspots.

Conclusions: The results indicate that if used in conjunction with a pre-treatment catheter displacement correction protocol and a tolerance of $3 \mathrm{~mm}$, a DTDC value of 0.4 may produce clinically superior plans. However, the effect of the DTDC parameter in plan robustness was not observed to be as strong as initially suspected.
\end{abstract}

Key words: brachytherapy, catheter displacement, DTDC, IPSA, prostate cancer.

\section{Purpose}

Prostate cancer is the most common male malignancy in the Western world, and as life expectancy increase, the prevalence is also expected to increase in an aging population [1]. Radiotherapy is an important therapeutic modality for the treatment of patients with localized or locally advanced prostate cancer [2] utilizing both external beam radiotherapy (EBRT) and brachytherapy. Over the last few decades, significant advances in technology related to high-dose-rate (HDR) brachytherapy have seen an increase in its use as a localized boost to EBRT of the prostate.

An important technological advancement in HDR brachytherapy is the evolution from forward planning to inverse planning techniques $[3,4,5]$. The inverse planning optimization algorithm currently implemented in the $\mathrm{Nu}-$ cletron Oncentra (Nucletron B.V., Veenendaal, The Netherlands) brachytherapy treatment planning system (TPS) is the inverse planning simulated annealing (IPSA) optimization algorithm. Inverse planning simulated annealing is based on contoured anatomy and optimizes dwell times using a simulated annealing algorithm [6]. The algorithm is constrained by user specific surface and volumetric dose constraints for both the target volume and organs at risk to calculate clinically acceptable treatment plans [7].

Inverse planning simulated annealing optimized brachytherapy treatment plans are characterized with large isolated dwell times at the first or last dwell position of each catheter. The central dwell positions however consist of extremely short, or zero, dwell times [8]. There is concern amongst users that these large isolated dwell times may lead to hot spots, either inside or outside the target. Also, the potential of catheter shifts relative to 
the target and organs at risk may lead to a more significant change in delivered dose to the volumes of interest relative to plans with more uniform dwell times. Recently, the Nucletron Oncentra TPS has added the dwell time deviation constraint (DTDC) parameter to the IPSA optimization process. This parameter constrains the allowable dwell times in the optimization process and can be set to a value between 0 and 1 in increments of 0.1 . A value of 0 corresponds to completely unrestricted dwell times and a value of 1 results in homogeneous dwell times [9].

The displacement of catheters relative to the target and organs at risk during the time between imaging and patient treatment has been reported by a number of groups $[10,11,12,13]$. The displacements have predominantly been reported along the patient longitudinal axis and in the caudal direction [10] primarily due to acute edema between the prostate and perineal skin [14]. Previous work from our group [11] demonstrated a median catheter displacement of $7.5 \mathrm{~mm}$ in caudal direction (range 2.9-23.9 mm) in the time from planning CT to treatment (approximately 1-3 hours). Tiong et al. [10] have reported significant adverse effects on the tumor control probability for catheter displacements larger than $3 \mathrm{~mm}$, including underdosage of the target and overdosage to critical structures. Due to these findings, our department has implemented a clinical protocol, in which internal catheter positions are verified and corrected immediately prior to treatment delivery with a tolerance of $3 \mathrm{~mm}$.

This study aims to determine if the DTDC parameter can be optimized to improve the robustness of HDR prostate brachytherapy plans to catheter displacements relative to patient anatomy. A set of 10 clinically acceptable prostate plans were re-optimized with a DTDC parameter of 0 and 0.4 . The values of 0 and 0.4 were chosen to reflect the change that is currently occurring in our clinical protocol. For each plan, catheter displacements of 3, 7, and $14 \mathrm{~mm}$ were retrospectively applied, and the change in DVH indices and conformity indices analyzed.

\section{Material and methods}

\section{Initial plans}

A set of 10 clinical prostate HDR brachytherapy plans were chosen for analysis. These CT plans were created between 2012 and 2015 on the Nucletron Oncentra Brachytherapy TPS (v4.3, Nucletron B.V., Veenendaal, The Netherlands). The prostate planning target volume (PTV), urethra, and rectum were all contoured by the same radiation oncologist at the time of treatment. Prostate volumes varied between 25.1 and $59.4 \mathrm{~cm}^{3}$ and the number of catheters used was between 14 and 24 . All patients received 2 fractions of 9.5 Gy with 2 weeks between fractions. Catheter insertion (using Oncosmart, ProGuide Sharp Needle, 6F, Nucletron B.V., Veenendaal, The Netherlands), CT scan, planning, and treatment are all performed on the same day, and the mean time between the planning CT and treatment was 182 minutes.

\section{IPSA optimization and DTDC}

Each plan was optimized using the IPSA algorithm using the parameters outlined in Table 1 . As per clinical protocol, plans were initially optimized with the DTDC parameter set to 0 . The plans were then re-optimized with the DTDC parameter set to 0.4 and all other parameters kept constant. The dwell time characteristics of each plan were then compared using the plan modulation index (M), as defined by Smith et al. [9]. The plan modulation index is defined as the maximum deviation of dwell time from the average dwell time for each catheter, normalized to the maximum dwell time for the treatment plan, averaged over all catheters in the plan.

\section{Catheter displacements}

Catheter displacements in the caudal direction were then simulated for each plan. Offsets of 3, 7, and $14 \mathrm{~mm}$ were performed. Displacements of this magnitude were chosen as they corresponded to clinically relevant catheter displacements, as found in a previous study by our group [11]. Our center has implemented a clinical protocol, in which catheter displacements $\geq 3 \mathrm{~mm}$ are corrected for, immediately prior to treatment by altering the indexer length at the treatment console. Implanting the catheters past the prostate base into the bladder allowed for extra dwell positions beyond the prostate in the event of a caudal shift. Physical re-insertion was not performed.

\section{Plan analysis}

All patient plans were assessed by evaluating dose volume histogram $(\mathrm{DVH})$ indices and dose quality indices. Dose volume histogram indices used for plan evaluation are outlined in Table 2. Furthermore, a normal tissue (NT) contour was created by adding a $2 \mathrm{~mm}$ margin around the PTV and subtracting this expanded contour from the external contour, e.g. NT = Body $-(\mathrm{PTV}+2 \mathrm{~mm})$. These parameters were automatically calculated by the

Table 1. Inverse planning simulated annealing (IPSA) optimization parameters used for patient plan optimization

\begin{tabular}{lccccccccc} 
ROI & Usage & \multicolumn{4}{c}{ Surface } & \multicolumn{3}{c}{ Volume } \\
\cline { 3 - 11 } & & Weight & Min (cGy) & Max (cGy) & Weight & Weight & Min (cGy) & Max (cGy) & Weight \\
\hline Prostate & PTV & 100 & 950 & 1425 & 100 & 100 & 100 & 950 & 30 \\
\hline Rectum & Organ & & & 665 & 50 & 50 & & 475 & 50 \\
\hline Urethra & Organ & 120 & 950 & 998 & 50 & 50 & 950 & 998 & 50
\end{tabular}

PTV - planning target volume 
TPS and are highly dependent on the size of the histogram bin used for calculation [8]. Because of this, a conformity index $(\mathrm{CI})$, a dose inhomogeneity index (DHI), and an overdose volume index (ODI) were also calculated for each plan.

The CI used in this study is the one introduced by van't Riet et al. [15] and is shown in Equation 1:

$\mathrm{CI}=\frac{\mathrm{V}_{\mathrm{T}, \mathrm{ref}}}{\mathrm{V}_{\mathrm{T}}} \times \frac{\mathrm{V}_{\mathrm{T}, \mathrm{ref}}}{\mathrm{V}_{\text {ref }}}$

where $V_{T, r e f}$ is the volume of the PTV receiving a dose greater than or equal to the $100 \%$ isodose, $V_{T}$ is the volume of the PTV, and $V_{\text {ref }}$ is the volume of the $100 \%$ isodose. The DHI parameter gives an indication of the homogeneity of the dose within the PTV, which was first introduced by Wu et al. [16] and is shown in Equation 2:

$\mathrm{DHI}=\frac{\mathrm{V}_{\mathrm{T}, \mathrm{ref}}-\mathrm{V}_{\mathrm{T}, 1.5 \mathrm{ref}}}{\mathrm{V}_{\mathrm{T}, \mathrm{ref}}}$

where $V_{T, 1.5 r e f}$ is the volume of the PTV receiving a dose greater than or equal to the $150 \%$ isodose, and $V_{T \text {,ref }}$ is as described above. Finally, the ODI parameter [17] indicates the amount of high dose (greater than 200\%) within the PTV:

$\mathrm{ODI}=\frac{\mathrm{V}_{\mathrm{T}, 2 \mathrm{ref}}}{\mathrm{V}_{\mathrm{T}, \mathrm{ref}}}$

where $V_{T, 2 \text { ref }}$ is the volume of the PTV receiving a dose greater than or equal to the $200 \%$ isodose.

The change in DVH and dose quality indices was then calculated as a function of catheter displacement for both DTDC values of 0 and 0.4. The change in these indices with increasing catheter displacement gives an indication of the robustness of the plans to changes in catheter po-

Table 3. Plan modulation index $(M)$ and normalized total dwell time $\left(\mathrm{cGy}^{-1} \mathrm{~cm}^{-2}\right)$

\begin{tabular}{lcccc} 
Patient & \multicolumn{2}{c}{$\begin{array}{c}\text { Plan modulation index } \\
(\mathrm{M})\end{array}$} & \multicolumn{2}{c}{$\begin{array}{c}\text { Normalized total dwell } \\
\text { time }\left(\mathrm{cGy}^{-1} \mathrm{~cm}^{-2}\right)\end{array}$} \\
\cline { 2 - 5 } & DTDC 0 & DTDC 0.4 & DTDC 0 & DTDC 0.4 \\
\hline 1 & 0.48 & 0.09 & $8.32 \times 10^{-6}$ & $7.73 \times 10^{-6}$ \\
\hline 2 & 0.34 & 0.23 & $2.75 \times 10^{-6}$ & $2.55 \times 10^{-6}$ \\
\hline 3 & 0.43 & 0.25 & $2.57 \times 10^{-6}$ & $2.33 \times 10^{-6}$ \\
\hline 4 & 0.41 & 0.23 & $10.44 \times 10^{-6}$ & $9.82 \times 10^{-6}$ \\
\hline 5 & 0.57 & 0.17 & $15.27 \times 10^{-6}$ & $14.32 \times 10^{-6}$ \\
\hline 6 & 0.43 & 0.18 & $12.45 \times 10^{-6}$ & $12.34 \times 10^{-6}$ \\
\hline 7 & 0.42 & 0.24 & $6.32 \times 10^{-6}$ & $6.12 \times 10^{-6}$ \\
\hline 8 & 0.48 & 0.19 & $8.72 \times 10^{-6}$ & $8.29 \times 10^{-6}$ \\
\hline 9 & 0.40 & 0.21 & $8.37 \times 10^{-6}$ & $7.80 \times 10^{-6}$ \\
\hline 10 & 0.44 & 0.20 & $7.27 \times 10^{-6}$ & $6.80 \times 10^{-6}$
\end{tabular}

DTDC - dwell time deviation constraint
Table 2. Clinically acceptable dose volume histogram (DVH) indices

\begin{tabular}{lccc} 
Volume type & Dose (\%) & Dose (cGy) & Volume (\%) \\
\hline PTV & 100 & 950 & $\geq 90$ \\
\hline PTV & 150 & 1425 & $<30$ \\
\hline PTV & 200 & 1900 & $<15$ \\
\hline Rectum & 70 & 665 & 0 \\
\hline Urethra & 120 & 1140 & 0
\end{tabular}

PTV - planning target volume

sition relative to the targets and organs at risk between planning CT and treatment. Statistical significance between the DTDC values was verified using a paired $t$-test with $\alpha=0.05$ (corresponding to a 5\% significance level).

\section{Results}

\section{Initial clinical plans}

Initial clinical prostate plans IPSA optimized with a DTDC value of 0 produced a large spread of dwell times, relative to those plans optimized with a DTDC value of 0.4. The plan modulation index (M) for each plan variant is given in Table 3 along with the total dwell time, normalized to the air kerma strength of the source. The average $M$ for the 0 DTDC case $( \pm 1 \mathrm{SD})$ was equal to $0.44 \pm 0.07$, whereas for the 0.4 DTDC case $M=0.20$ \pm 0.06 . The effect of increasing the DTDC parameter is to limit the maximum dwell time in any catheter; this is reflected by the decreasing value of $\mathrm{M}$, as more homogeneous dwell time distribution is created within each cath-

Table 4. Initial dose volume histogram (DVH) and dose indices before catheter displacement

\begin{tabular}{lccc} 
Parameter & DTDC 0 & DTDC 0.4 & $p$ \\
\hline PTV $V_{100 \%}$ & $92.9 \pm 1.9 \%$ & $93.4 \pm 1.7 \%$ & $\leq 0.2620$ \\
\hline PTV $V_{150 \%}$ & $18.9 \pm 3.6 \%$ & $23.0 \pm 3.7 \%$ & $\leq 0.0001$ \\
\hline PTV $_{200 \%}$ & $7.5 \pm 1.6 \%$ & $9.0 \pm 1.4 \%$ & $\leq 0.0002$ \\
\hline Rectum $V_{70 \%}$ & $0.1 \pm 0.1 \%$ & $0.1 \pm 0.1 \%$ & $\leq 0.3498$ \\
\hline Urethra $V_{120 \%}$ & $10.4 \pm 5.6 \%$ & $5.9 \pm 5.9 \%$ & $\leq 0.0421$ \\
\hline NT $V_{100 \%}$ & $11.0 \pm 3.0 c c$ & $5.7 \pm 1.9 c c$ & $\leq 0.0001$ \\
\hline NT $V_{150 \%}$ & $3.3 \pm 1.4 c c$ & $1.2 \pm 0.7 c c$ & $\leq 0.0001$ \\
\hline NT $V_{200 \%}$ & $1.5 \pm 0.2 c c$ & $0.5 \pm 0.3 c c$ & $\leq 0.0018$ \\
\hline Cl & $0.691 \pm 0.046$ & $0.748 \pm 0.042$ & $\leq 0.0002$ \\
\hline DHI & $0.204 \pm 0.039$ & $0.246 \pm 0.038$ & $\leq 0.0001$ \\
\hline ODI & $0.081 \pm 0.018$ & $0.097 \pm 0.014$ & $\leq 0.0004$
\end{tabular}

PTV - planning target volume, NT - normal tissue, Cl - conformity index, $D H I$ - dose inhomogeneity index, ODI - overdose index, $V_{100 \%}, V_{150 \%}, V_{200 \%}$ $V_{70 \%}, V_{120 \%}$ - volume of relevant structure receiving $100 \%, 150 \%, 200 \%, 70 \%$, and $120 \%$ of the prescribed isodose, respectively, DTDC - dwell time deviation constraint 
Table 5. Change in dose volume histogram (DVH) and dose indices for a $3 \mathrm{~mm}$ catheter displacement

\begin{tabular}{lccc} 
Parameter & DTDC 0 & DTDC 0.4 & $p$ \\
\hline PTV $V_{100 \%}$ & $0.788 \pm 0.751 \%$ & $-1.299 \pm 0.916 \%$ & $\leq 0.0001$ \\
\hline PTV $V_{150 \%}$ & $1.786 \pm 1.540 \%$ & $0.146 \pm 0.537 \%$ & $\leq 0.0009$ \\
\hline PTV $V_{200 \%}$ & $1.051 \pm 0.829 \%$ & $-0.034 \pm 0.183 \%$ & $\leq 0.0021$ \\
\hline Rectum $V_{70 \%}$ & $0.078 \pm 0.081 \%$ & $0.080 \pm 0.090 \%$ & $\leq 0.8905$ \\
\hline Urethra $V_{120 \%}$ & $-5.628 \pm 4.878 \%$ & $-4.019 \pm 5.047 \%$ & $\leq 0.0088$ \\
\hline NT $V_{100 \%}$ & $-1.042 \pm 1.314 c c$ & $1.314 \pm 1.661 c c$ & $\leq 0.0454$ \\
\hline NT $V_{150 \%}$ & $-1.033 \pm 0.893 c c$ & $0.468 \pm 0.504 c c$ & $\leq 0.0001$ \\
\hline NT $V_{200 \%}$ & $-0.568 \pm 0.674 c c$ & $0.171 \pm 0.318 c c$ & $\leq 0.0052$ \\
\hline Cl & $0.002 \pm 0.017$ & $-0.024 \pm 0.022$ & $\leq 0.0011$ \\
\hline DHI & $0.017 \pm 0.005$ & $0.005 \pm 0.006$ & $\leq 0.0033$ \\
\hline ODI & $0.011 \pm 0.008$ & $0.001 \pm 0.002$ & $\leq 0.0035$
\end{tabular}

PTV - planning target volume, NT - normal tissue, Cl - conformity index $\mathrm{DHI}$ - dose inhomogeneity index, ODI - overdose index, $V_{100 \%}, V_{150 \%}, V_{200 \%}$ $V_{70 \%}, V_{120 \%}$ - volume of relevant structure receiving $100 \%, 150 \%, 200 \%, 70 \%$, and $120 \%$ of the prescribed isodose, respectively, DTDC - dwell time deviation constraint

eter. The total dwell time, normalized to the air kerma strength of the source was also seen to decrease for plans optimized with a DTDC value of 0.4 , relative to a DTDC of 0 . This is due to the reduction in large isolated dwell times at the first or last dwell positions of each catheter.

Table 4 shows the change in DVH and dose quality indices when re-optimizing the plans with a DTDC parameter of 0.4. By changing the DTDC value to 0.4,

Table 6. Change in dose volume histogram (DVH) and dose indices for a $7 \mathrm{~mm}$ catheter displacement

\begin{tabular}{lccc} 
Parameter & DTDC 0 & DTDC 0.4 & $p$ \\
\hline PTV $V_{100 \%}$ & $-2.803 \pm 2.516 \%$ & $-8.99 \pm 2.283 \%$ & $\leq 0.0001$ \\
\hline PTV $V_{150 \%}$ & $2.199 \pm 2.670 \%$ & $-1.262 \pm 1.158 \%$ & $\leq 0.0002$ \\
\hline PTV $V_{200 \%}$ & $1.255 \pm 1.260 \%$ & $-0.689 \pm 0.427 \%$ & $\leq 0.0008$ \\
\hline Rectum $V_{70 \%}$ & $0.271 \pm 0.324 \%$ & $0.265 \pm 0.324 \%$ & $\leq 0.7793$ \\
\hline Urethra $V_{120 \%}$ & $-6.461 \pm 5.879 \%$ & $-4.333 \pm 6.804 \%$ & $\leq 0.0205$ \\
\hline NT $V_{100 \%}$ & $1.072 \pm 3.121 c c$ & $4.381 \pm 1.996 c c$ & $\leq 0.0189$ \\
\hline NT $V_{150 \%}$ & $-0.399 \pm 1.639 c c$ & $1.507 \pm 0.753 c c$ & $\leq 0.0041$ \\
\hline NT $V_{200 \%}$ & $-0.471 \pm 0.940 c c$ & $0.641 \pm 0.517 c c$ & $\leq 0.0014$ \\
\hline CI & $-0.046 \pm 0.044$ & $-0.136 \pm 0.046$ & $\leq 0.0005$ \\
\hline DHI & $0.029 \pm 0.024$ & $0.011 \pm 0.016$ & $\leq 0.0007$ \\
\hline ODI & $0.016 \pm 0.012$ & $0.002 \pm 0.004$ & $\leq 0.0020$
\end{tabular}

PTV - planning target volume, NT - normal tissue, $\mathrm{Cl}$ - conformity index, $\mathrm{DHI}$ - dose inhomogeneity index, ODI - overdose index, $V_{100 \%}, V_{150 \%}, V_{200 \%}$ $V_{70 \%}, V_{120 \%}$ - volume of relevant structure receiving $100 \%, 150 \%, 200 \%, 70 \%$, and $120 \%$ of the prescribed isodose, respectively, DTDC - dwell time deviation constraint the coverage of the PTV is improved, as reflected by the increase in PTV $\mathrm{V}_{100 \%}$ and CI, however, only the difference in CI was found to be statistically significant. Statistically significant reductions in $\mathrm{NT} \mathrm{V}_{100 \%}, \mathrm{NT} \mathrm{V}_{150 \%}$, and NT $V_{200 \%}$ were also found when changing DTDC from 0 to 0.4 . This, along with the improvement in $\mathrm{CI}$, is due to the reduction in the large isolated dwell times just outside of the PTV, which are delivering higher doses to the adjacent healthy tissue. On the other hand, there is a statistically significant increase in PTV $\mathrm{V}_{150 \%}$, PTV V $200 \%$, DHI, and ODI for DTDC 0.4 versus DTDC 0 . The rectum $\mathrm{V}_{150 \%}$ was largely unaffected by the DTDC change, and a small reduction in the urethra $\mathrm{V}_{120 \%}$ was observed.

\section{Effect of catheter displacements}

The effect of catheter displacements on DVH and dose quality indices are shown in Tables 5, 6, and 7 for catheter shifts of 3,7, and $14 \mathrm{~mm}$, respectively. Overall, a DTDC value of 0 improves the robustness of PTV coverage to catheter displacements relative to a DTDC value of 0.4. This is reflected in the smaller changes in PTV $V_{100 \%}$ (Figure 1) and CI (Figure 2) for all three catheter displacement values. The dwell positions moving out of the PTV in the DTDC 0 plans have smaller weights relative to those in the DTDC 0.4 plans, resulting in smaller changes in PTV V $100 \%$ and CI with catheter displacement.

Conversely, a DTDC value of 0.4 improves the robustness of the plans to changes in hotspots, reflected by statistically significant differences in changes to PTV $\mathrm{V}_{150 \%}$, PTV V $200 \%$ (Figure 3), DHI, and ODI (Figure 4) compared to plans optimized with a DTDC value of 0 for catheter displacements up to $14 \mathrm{~mm}$. This behavior can be explained by considering that the isolated dwell times at the end of the catheters often exist just outside the PTV be-

Table 7. Change in dose volume histogram (DVH) and dose indices for a $14 \mathrm{~mm}$ catheter displacement

\begin{tabular}{lccc} 
Parameter & DTDC 0 & DTDC 0.4 & $p$ \\
\hline PTV $V_{100 \%}$ & $-18.69 \pm 5.197 \%$ & $-25.74 \pm 5.313 \%$ & $\leq 0.0003$ \\
\hline PTV $V_{150 \%}$ & $0.522 \pm 3.343 \%$ & $-3.589 \pm 2.063 \%$ & $\leq 0.0001$ \\
\hline PTV $V_{200 \%}$ & $0.566 \pm 1.455 \%$ & $-1.628 \pm 0.786 \%$ & $\leq 0.0005$ \\
\hline Rectum $V_{70 \%}$ & $0.690 \pm 0.917 \%$ & $0.665 \pm 0.956 \%$ & $\leq 0.4049$ \\
\hline Urethra $V_{120 \%}$ & $-4.657 \pm 7.543 \%$ & $-0.592 \pm 10.624 \%$ & $\leq 0.0523$ \\
\hline NT $V_{100 \%}$ & $8.928 \pm 4.198 c c$ & $13.495 \pm 2.333 c c$ & $\leq 0.0002$ \\
\hline NT $V_{150 \%}$ & $1.582 \pm 1.210 c c$ & $3.981 \pm 1.197 c c$ & $\leq 0.0008$ \\
\hline NT $V_{200 \%}$ & $0.453 \pm 0.786 c c$ & $1.756 \pm 0.747 c c$ & $\leq 0.0036$ \\
\hline Cl & $-0.251 \pm 0.072$ & $-0.352 \pm 0.064$ & $\leq 0.0003$ \\
\hline DHI & $0.056 \pm 0.040$ & $0.041 \pm 0.039$ & $\leq 0.0013$ \\
\hline ODI & $0.027 \pm 0.018$ & $0.013 \pm 0.010$ & $\leq 0.0043$
\end{tabular}

PTV - planning target volume, NT - normal tissue, CI - conformity index, DHI - dose inhomogeneity index, ODI - overdose index, $V_{100 \%,} V_{150 \%}, V_{200 \%}$, $V_{70 \%}, V_{120 \%}$ - volume of relevant structure receiving $100 \%, 150 \%, 200 \%, 70 \%$, and $120 \%$ of the prescribed isodose, respectively, DTDC - dwell time deviation constraint 


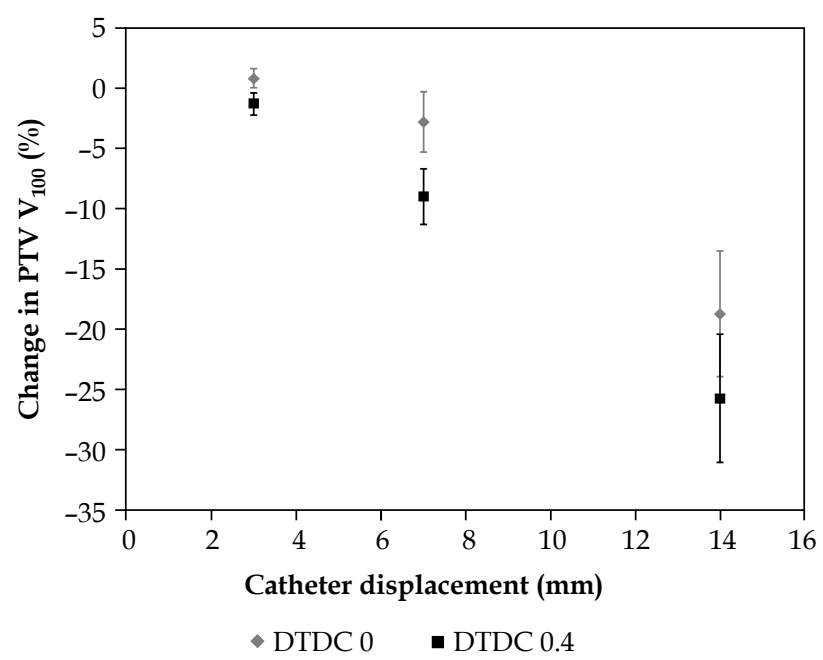

Fig. 1. The change in PTV $V_{100 \%}$ as a function of catheter displacement for plans optimized with DTDC set to 0 and 0.4 (error bars showing 95\% confidence interval)

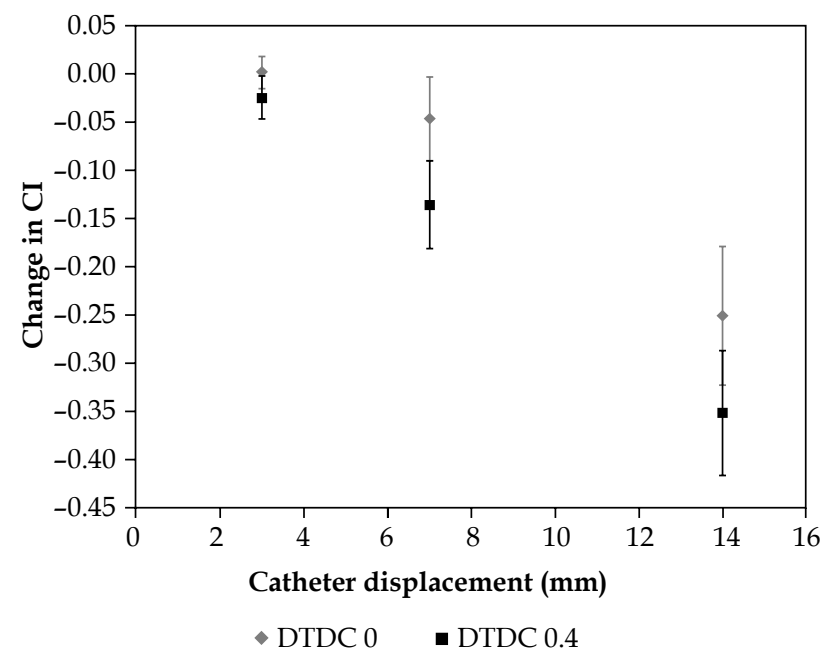

Fig. 3. The change in $\mathrm{CI}$ as a function of catheter displacement for plans optimized with DTDC set to 0 and 0.4 (error bars showing 95\% confidence interval)

fore a catheter shift is implemented. Therefore, the $\mathrm{V}_{150 \%}$ and $V_{200 \%}$ volumes are surrounding these dwell positions and, as a catheter shift is implemented, they move further away from the PTV and into healthy tissue.

For a catheter displacement of $3 \mathrm{~mm}$, plans optimized with a DTDC value of 0.4 were found to be more robust in terms of NT $\mathrm{V}_{100 \%}, \mathrm{NT} \mathrm{V}_{150 \%}$, and NT V $200 \%$. Conversely, for larger catheter shifts of 7 and $14 \mathrm{~mm}$, the plans optimized with DTDC 0 were more robust. This is due to the fact that for a catheter shift of $3 \mathrm{~mm}$, one of the large isolated dwell positions in the DTDC 0 plans moves into the normal tissue. However, for larger shifts, subsequent dwell positions moving into the normal tissue have significantly smaller dwell times compared to those in the DTDC 0.4 plans, resulting in smaller changes in $\mathrm{NT} \mathrm{V}_{100 \%}$, NT V $150 \%$, and NT $V_{200 \%}$.

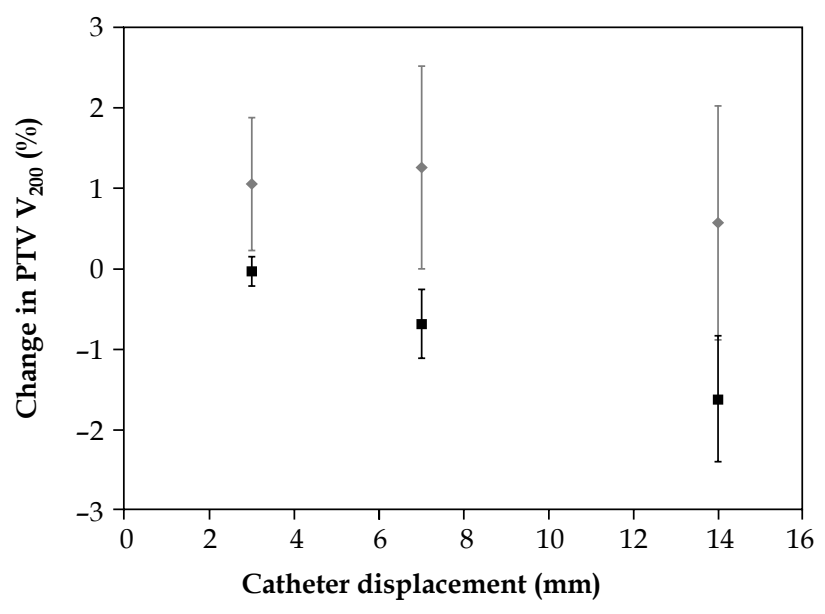

- DTDC 0 - DTDC 0.4

Fig. 2. The change in PTV $V_{200 \%}$ as a function of catheter displacement for plans optimized with DTDC set to 0 and 0.4 (error bars showing $95 \%$ confidence interval)

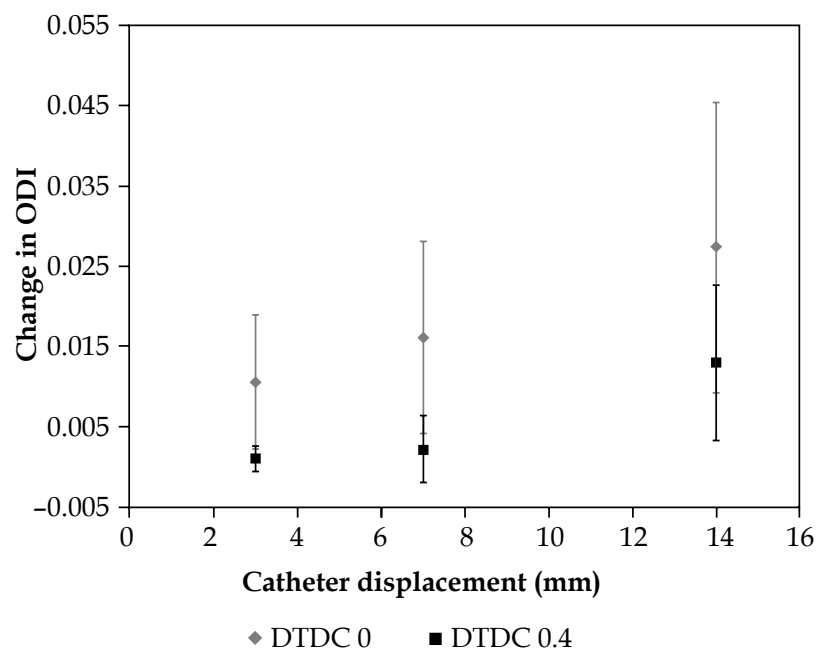

Fig. 4. The change in ODI as a function of catheter displacement for plans optimized with DTDC set to 0 and 0.4 (error bars showing 95\% confidence interval)

The urethra $\mathrm{V}_{120 \%}$ was more sensitive to catheter displacements than the rectum $\mathrm{V}_{70 \%}$ for both values of DTDC. A DTDC value of 0.4 improved the robustness of the plans to changes in urethra $\mathrm{V}_{120 \%}$ compared to plans optimized with a DTDC value of 0 , with statistically significant differences for catheter displacements of 3 and $7 \mathrm{~mm}$. There was also no statistically significant difference found between DTDC values for the rectum $V_{70 \%}$.

\section{Discussion}

As expected, the plan modulation index (M) was observed to decrease with an increased value of DTDC. The calculated values of $\mathrm{M}=0.44 \pm 0.07(\mathrm{DTDC}=0)$ and $\mathrm{M}=0.20 \pm 0.06(\mathrm{DTDC}=0.4)$ are in close agreement with those found in a previous study by Smith et al. [9]. The in- 
crease value of DTDC was also observed to increase the PTV $\mathrm{V}_{100 \%}, \mathrm{~V}_{150 \%}$, and $\mathrm{V}_{200 \%}$. The increase in PTV $\mathrm{V}_{150 \%}$ of $4 \%$ and PTV $V_{200 \%}$ of $1.5 \%$ when changing the DTDC value from 0 to 0.4 was also observed in the same study [9]. This increase resulted in DVH indices that were still clinically acceptable for treatment according to our local protocol as outlined in Table 2.

Contrary to expectation, the DTDC value of 0 produced plans that were more robust to catheter displacements in terms of target coverage. However, for a catheter displacement of $3 \mathrm{~mm}$, the average CI was $0.693 \pm 0.049$ and $0.723 \pm 0.045$ for DTDC $=0$ and DTDC $=0.4$, respectively. Our center has implemented a clinical protocol, in which catheter displacements $\geq 3 \mathrm{~mm}$ are corrected for, immediately prior to treatment by adjusting the indexer length on the treatment control system. Therefore, if used in combination with this catheter correction protocol, plans optimized with a DTDC value of 0.4 may be clinically superior to those optimized with DTDC $=0$, especially when considering that using a DTDC value of 0.4 improves robustness to changes in hotspots and dose to OARs and healthy tissue.

One recent advance in the field of HDR prostate brachytherapy has been the use of 3D trans-rectal ultrasound (TRUS) based treatment planning [18]. The use of this technique has been shown to significantly reduce the time between imaging and treatment compared to CT based treatment planning. Milickovic et al. [18] have shown that for an average time between imaging and treatment of 51.2 minutes, the average needle displacement was found to be $1 \mathrm{~mm}$. This displacement is small relative to those noted in other studies $[11,14]$, and is likely due to the reduction in time between imaging and treatment. Therefore, one current initiative of our group is to reduce the time between imaging and treatment, and the introduction of 3D TRUS based planning is being investigated.

A previous study by our group [11] has shown that catheter displacements in the cranial direction occurred for only 3 of 48 cases, with the remainder occurring in the caudal direction. One limitation of this study is that catheter displacements were only considered along the longitudinal axis in the caudal direction. Catheter shifts in the lateral and anterior-posterior directions due to edema were also not considered.

A further limitation is that only two values of DTDC were considered. Preliminary calculations showed that small changes in DTDC, e.g. from 0.2-0.4 do not significantly affect the robustness of the plans to catheter displacements. Furthermore, values of 0 and 0.4 were chosen to reflect the change that is currently occurring in our clinical protocol. Historically, plans have been optimized using a DTDC value of 0 ; however previous studies [9] have shown that a DTDC value of 0.4 gives plan modulation equivalent to graphical optimization without significantly compromising plan quality.

\section{Conclusions}

The results of this study indicate that the robustness of clinically acceptable prostate plans to catheter displace- ments in the caudal direction are dependent on the DTDC parameter. A DTDC value of 0 improves the robustness of PTV coverage to catheter displacements relative to a DTDC value of 0.4 . Whereas a DTDC value of $0.4 \mathrm{im}-$ proves the robustness of the plans to changes in hotspots compared to a DTDC value of 0 . For a catheter displacement of $3 \mathrm{~mm}$, plans optimized with a DTDC value of 0.4 were found to be more robust in terms of the dose to normal tissue. However, for larger catheter shifts, the plans optimized with DTDC 0 were more robust, due to larger shifts moving relatively small weight dwell positions into the normal tissue compared to the DTDC 0.4 plans. When used in combination with a pre-treatment catheter displacement correction protocol and a tolerance of $3 \mathrm{~mm}$, a DTDC value of 0.4 may produce clinically superior plans.

In future work, attempts will be made to measure the actual dwell times delivered by the afterloader as compared to those calculated by the TPS for a range of DTDC values and the effect on the dose examined.

\section{Acknowledgments}

The authors would like to acknowledge Jon Whitaker for his assistance with statistical analysis of the collected data and Ryan Smith for his useful discussion on the IPSA optimization algorithm and DTDC parameter.

\section{Disclosure}

Authors report no conflict of interest.

\section{References}

1. Quon H, Loblaw A, Nam R. Dramatic increase in prostate cancer cases by 2021. BJU International 2011; 108: 1734-1738.

2. Mangar SA, Huddart RA, Parker CC et al. Technological advances in radiotherapy for the treatment of localised prostate cancer. Eur J Cancer 2005; 41: 908-921.

3. Lessard E. Pouliot J. Inverse planning anatomy-based dose optimization for HDR-brachytherapy of the prostate using fast simulated annealing algorithm and dedicated objective function. Med Phys 2001; 28: 773-779.

4. Lachance B, Béliveau-Nadeau D, Lessard E et al. Early clinical experience with anatomy-based inverse planning dose optimization for high-dose-rate boost of the prostate. Int J Radiat Oncol Biol Phys 2002; 54: 86-100.

5. Karabis A, Belotti P, Baltas D. Optimization of Catheter Position and Dwell Time in Prostate HDR Brachytherapy using HIPO and Linear Programming. World Congress on Medical Physics and Biomedical Engineering 2009; 612-615.

6. Kirkpatrick S, Gelatt CD, Vecchi MP. Optimization by Simulated Annealing. Science 1983; 220: 671-680.

7. Dinkla AM, van der Laase R, Kajouw E et al. A comparison of inverse optimization algorithms for HDR/PDR prostate brachytherapy treatment planning. Brachytherapy 2015; 14 : 279-288.

8. Panettieri V, Smith RL, Mason NJ et al. Comparison of IPSA and HIPO inverse planning optimization algorithms for prostate HDR brachytherapy. J Appl Clin Med Phys 2014; 15: 256-266.

9. Smith R, Panettieri V, Lancaster C et al. The influence of the dwell time deviation constraint (DTDC) parameter on dosimetry with IPSA optimisation for HDR prostate brachytherapy. Austral Phys Eng Scienc Med 2015; 381: 55-61. 
10. Tiong A, Bydder S, Ebert M et al. A Small Tolerance for Catheter Displacement in High-Dose Rate Prostate Brachytherapy is Necessary and Feasible. Int J Radiat Oncol Biol Phys 2010; 76: 1066-1072.

11. Whitaker M, Hruby G, Lovett A et al. Prostate HDR brachytherapy catheter displacement between planning and treatment delivery. Radiother Oncol 2011; 101: 490-494.

12. Kim Y, Hsu I-CJ, Pouliot J. Measurement of cranio-caudal catheter displacement between fractions in CT-based HDR brachytherapy of prostate cancer. J Appl Clin Med Phys 2007; 8: $1-13$

13. Holly R, Morton GC, Sankreacha R et al. Use of cone-beam imaging to correct for catheter displacement in high doserate prostate brachytherapy. Brachytherapy 2011; 10: 299-305.

14. Hoskin PJ, Bownes PJ, Ostler P et al. High dose rate afterloading brachytherapy for prostate cancer: catheter and gland movement between fractions. Radiother Oncol 2003; 68: 285-258.

15. van't Riet A, Mak AC, Moerland MA et al. A conformation number to quantify the degree of conformality in brachytherapy and external beam irradiation: Application to the prostate. Int J Radiat Oncol Biol Phys 1997; 37: 731-736.

16. Wu A, Ulin K, Sternick ES. A dose homogeneity index for evaluating 192Ir interstitial breast implants. Med Phys 1988; 15: 104-107.

17. Anbumani S, Anchineyan P, Narayanasamy S et al. Treatment Planning Methods in High Dose Rate Interstitial Brachytherapy of Carcinoma Cervix: A Dosimetric and Radiobiological Analysis. ISRN Oncology 2014; 2014: 1-5.

18. Milickovic N, Mavroidis P, Tselis N et al. 4D analysis of influence of patient movement and anatomy alteration on the quality of 3D U/S-based prostate HDR brachytherapy treatment delivery. Med Phys 2011; 38: 4982-4993. 